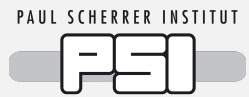

\section{The pion beta and radiative electronic decays}

\author{
D. Počanić ${ }^{1 \star}$, for the PiBeta Collaboration
}

1 University of Virginia, Charlottesville, VA 22904-4714, USA

*pocanic@virginia.edu

11 May 2021

Review of Particle Physics at PSI

doi:10.21468/SciPostPhysProc.2

\title{
Abstract
}

8 As the lightest meson, pion offers unique opportunities for measuring parameters and 9 testing limits of the Standard Model (SM). The PiBeta experiment, carried out at PSI, focused on SM tests accessible through the pion beta, $\pi^{+} \rightarrow \pi^{0} e^{+} v_{e}(\gamma)$, and electronic 1 radiative, $\pi^{+} \rightarrow e^{+} v_{e} \gamma$, decay channels. We review the PiBeta experiment, and update the 12 pion beta decay branching ratio $B_{\pi \beta}^{\exp }=1.038(6)_{\text {tot }} \times 10^{-8}$, along with the corresponding derived value of the Cabibbo-Kobayashi-Maskawa matrix element $V_{u d}=0.9738(28)$.

\subsection{Motivation}

Since $\left|V_{\mathrm{ud}}\right|^{2} \approx 0.95$ dominates $\left|V_{\mathrm{u}}\right|^{2}$, the uncertainty $\Delta V_{\mathrm{ud}}$ is critically important in evaluating $\Delta_{\text {CKM }}$. In spite of notable improvements in measurement and theoretical precision since the 1980 s, a shortfall of $\Delta_{\text {СKM }} \sim-3 \sigma$ has persisted for much of the past three decades. The discovery potential inherent in precision tests of CKM unitarity has motivated a worldwide effort. A summary of the present status of CKM unitarity tests is given in [1]. The most precise evaluations of $V_{\mathrm{ud}}$ have relied on the $0^{+} \rightarrow 0^{+}$superallowed Fermi (SAF) nuclear beta decays (for the most recent compilation see [2]). Despite the impressive experimental precision achieved in determining SAF $f t$ values, uncertainties related to the complex structure of participating nuclei remain, motivating the quest for $V_{\mathrm{ud}}$ evaluation in beta decays of simpler systems: neutrons and pions. Of the two, the pion beta semileptonic decay $\pi^{+} \rightarrow \pi^{0} e^{+} v_{e}(\gamma)^{1}$, or $\pi_{e 3(\gamma)}$, is the theoretically cleanest [3]. Given the small accessible phase space, $\pi_{e 3}$ decay is very rare: $B_{\pi \beta} \simeq 10^{-8}$. Neutron beta decay is not suppressed, but requires two measurements for an independent determination of $V_{\mathrm{ud}}$ : the lifetime, $\tau_{n}$, and the axial-vector coupling, $g_{A}=G_{A} / G_{V}$, (for further details and current status see [1,4-6]).

The international PiBeta collaboration [7], led by the University of Virginia group, was formed in the 1990s with the goal of measuring the pion beta decay branching ratio to a

\footnotetext{
${ }^{1} \mathrm{~A} \gamma$ in parentheses denotes an undetected, usually soft photon. For brevity, in further text the $(\gamma)$ will be dropped and implied; a detected photon in radiative processes will be explicitly denoted with a $\gamma$.
} 
precision of $0.5 \%$ at the Paul Scherrer Institute. Achieving this goal also requires accurate identification and detection of background and normalization decays: pion radiative electronic $\pi^{+} \rightarrow e^{+} v_{e} \gamma$, or $\pi_{e 2 \gamma}$, pion electronic $\pi^{+} \rightarrow e^{+} v_{e}$, or $\pi_{e 2}$, radiative muon $\mu^{+} \rightarrow e^{+} v_{e} \bar{v}_{\mu} \gamma$, and ordinary muon $\mu^{+} \rightarrow e^{+} v_{e} \bar{v}_{\mu}$ decay. Each of these processes illuminates interesting aspects of $\mathrm{SM} / \mathrm{BSM}$ physics. Muon decays will not be discussed here, while the electronic, $\pi_{e 2}$ decay is discussed in more detail in [8].

Unlike its muonic equivalent $\pi_{\mu 2 \gamma}$, the radiative electronic decay, $\pi_{e 2 \gamma}$, is not completely dominated by purely electromagnetic (QED) "inner" bremsstrahlung (IB). It also receives strong "structure-dependent" (SD) QCD contributions, parameterized in terms of $F_{V}$ and $F_{A}$, the vector and the axial-vector form factors, respectively. Direct determination of $F_{A, V}$ is possible through a precise measurement of the differential branching ratio, or decay rate $\mathrm{d}^{2} \Gamma_{\pi e 2 \gamma} / \mathrm{d} E_{e} \mathrm{~d} E_{\gamma}$, over a suitably large portion of the decay phase space $[9,10]$. Precise values of $F_{A}$ and $F_{V}$ provide information on nonperturbative QCD, such as the pion polarizabilities, and generally enter certain low energy (chiral) constants, LECs (for more details see, e.g., $[11,12])$. On the other hand, a kinematically broad sample of $\pi_{e 2 \gamma}$ decays makes it possible to set limits on values of form factors other than $F_{A, V}$, that, if nonzero, would indicate presence of BSM particles or processes. PiBeta has pursued both of these research paths, as discussed below.

\subsection{The PiBeta apparatus}

The PiBeta apparatus, schematically shown in Figure 24.1, detected $\pi^{+}$decays at rest in a solid cylindrical active target (AT), placed at the center of a pure CsI, 240-element spherical electromagnetic shower calorimeter. Prior to stopping, pions passed through a pair of scintillation detectors (BC and $\mathrm{AD}$ ) separated by a $\sim 3.5 \mathrm{~m}$ flight path. The segmented target was surrounded by two MWPC tracking detectors, and a fast 20-element hodoscope, shown schematically in Figure 24.2. The apparatus acquired data during a " $\pi$-stop" gate spanning $t \simeq-50$ to $200 \mathrm{~ns}$ relative to a pion stop time $(t=0)$ in the target, with a break of $\sim 10 \mathrm{~ns}$ at

CALO: pure CsI 240-element calorimeter BC: upstream Beam Counter AC1,2: Active beam Collimators AD: Active Degrader detector AT: Active Target detector MWPC1,2: Multi-Wire Proportional Chambers PH: Plastic Hodoscope, 20 staves (MWPCs, $\mathrm{PH}$ have cylindical shape)

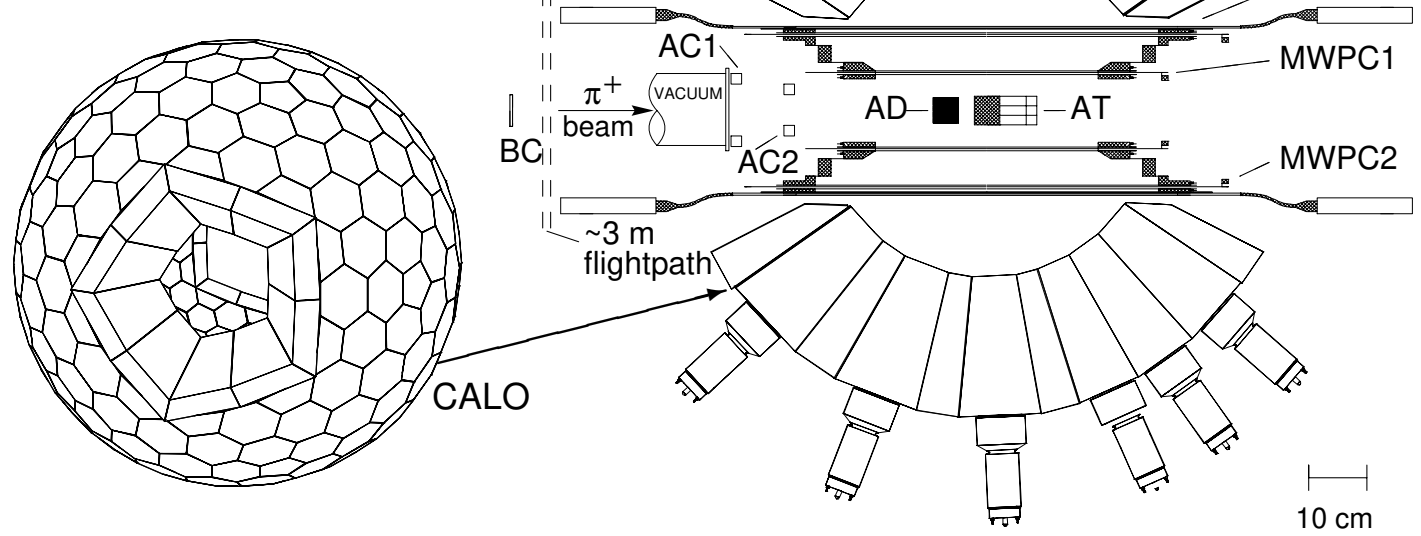

Figure 24.1: Schematic cross section of the PiBeta apparatus, with its main components labeled. For details concerning the detector performance see [13]. 


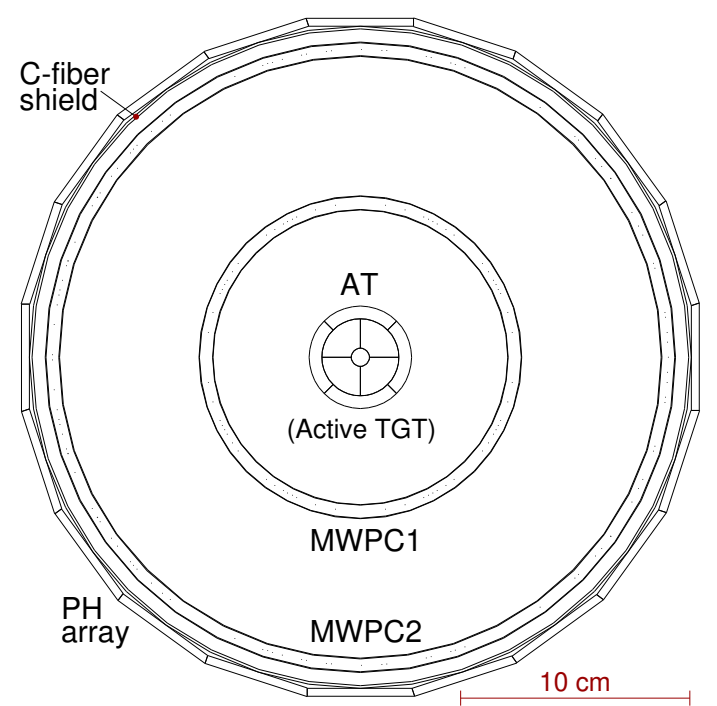

Figure 24.2: Axial (beam) view of the central detector region used in PiBeta Runs 1-3, and first half of Run 4. Outward from center: (i) the 9-element segmented active target AT, (ii) cylindrical MWPC1 and MWPC2 trackers, (iii) thin cylindrical carbon-fiber shield around MWPC2, and (iv) the 20-element plastic hodoscope (PH) array with approximate outer diameter of $\varnothing 30 \mathrm{~cm}$. Pion stopping rates in the inner five (fiducial) target elements were roughly matched; AT outer ring elements served for decay particle tracking. The BC, $\mathrm{AD}, \mathrm{AT}$ and $\mathrm{PH}$ detectors were made of fast plastic scintillator.

$t=0$ because of high rates of hadronic reactions by beam pions in $\mathrm{AD}$ and AT. The calorimeter modules were sized such that, on average, a crystal impacted centrally by a $70 \mathrm{MeV} e^{+}$or $\gamma$ would contain over $90 \%$ of the resulting shower energy. The location and energy of each distinct shower in an event were extracted for trigger purposes from continuous analog signal sums of overlapping clusters of 7-9 modules. A dozen trigger configurations, combining calorimeter and beam detector hit patterns of interest, were used to acquire the studied and normalization decay events, as well as all relevant background processes. Further details of the design and performance of the apparatus are given in [13]. For a discussion of the PiBeta technique in a broader context, see [14].

\subsection{The pion beta decay: $\pi^{+} \rightarrow \pi^{0} e^{+} v_{e}(\gamma)$}

PiBeta measurements were carried out in four run periods, using $114 \mathrm{MeV} / \mathrm{c}$ beam in the $\pi \mathrm{E} 1$ beamline at PSI. Over $6.4 \times 10^{4} \pi_{e 3}$ events were acquired in high-rate Runs 1-3 (1999-2001), with $\sim 10^{6} \pi_{\text {stop }}^{+} / \mathrm{s}$ in the target. Run 4 (2004), with $10^{4}-10^{5} \pi_{\text {stop }}^{+} / \mathrm{s}$ in the target, focused on the radiative decay $\pi_{e 2 \gamma}$. The $\pi_{e 3}$ decay signal, two energetic, nearly back to back neutral showers in the calorimeter, initiated by the two photons from $\pi^{0} \rightarrow \gamma \gamma$ decay, is robust and required minimal background subtractions. Figure 24.3 illustrates the quality of the PiBeta $\pi_{e 3}$ event sample. The $\theta_{\gamma 1 \gamma 2}$ distribution, uniquely shaped by the decay kinematics and the shower
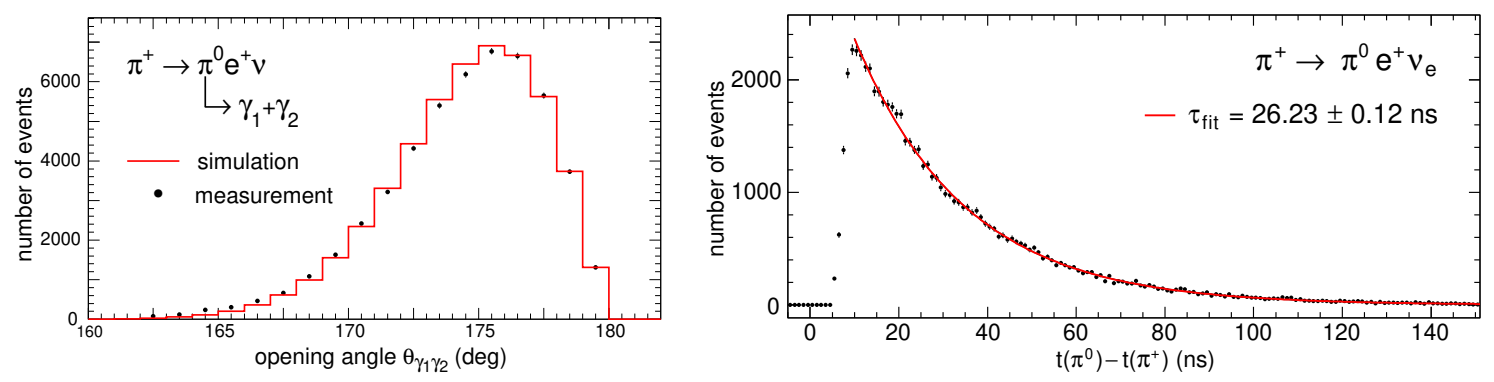

Figure 24.3: Left: measured photon-photon opening angle in $\pi^{0} \rightarrow \gamma \gamma$, compared to a realistic Monte Carlo simulation. Right: decay time distribution for $\pi_{e 3}$ events. Events within $\sim 10 \mathrm{~ns}$ of the $\pi^{+}$stop in AT were not recorded due to high prompt hadronic background. 
response of the calorimeter, is not reproduced in other processes. The decay time distribution is purely exponential, and agrees well with the known pion lifetime of 26.033(5) ns [1]. The $\pi^{+} \rightarrow e^{+} v_{e}(\gamma)$ electronic decay events were used for branching ratio normalization. While the two decays shared many of the same systematics, such as the spatial and temporal distributions of the parent pions, and very similar acceptances, the $\pi_{e 2}$ signal had a significant background from the "Michel" $\mu^{+} \rightarrow e^{+} v \bar{v}(\gamma)$ decays. Details of the analysis and results for the $\pi_{e 3}$ branching ratio are discussed in [15]. Two values of $B_{\pi \beta}=\Gamma\left(\pi^{+} \rightarrow \pi^{0} e^{+} v(\gamma)\right) / \Gamma\left(\pi^{+} \rightarrow \mu^{+} \nu(\gamma)\right)$ were evaluated and reported: one normalized to the accepted 2004 experimental average of $R_{e / \mu}^{\pi \text {-exp }}=\Gamma(\pi \rightarrow e \bar{v}(\gamma)) / \Gamma(\pi \rightarrow \mu \bar{v}(\gamma))=1.230(4) \times 10^{-4}$ ("exp-norm"), and the second to the established theoretical value $R_{e / \mu}^{\pi \text {-th }}=1.2352(5) \times 10^{-4}$ ("theo-norm"):

$$
\begin{gathered}
B_{\pi \beta}^{\text {exp-norm }}=1.036(4)_{\text {stat }}(4)_{\text {syst }}(3)_{\pi_{e 2}} \times 10^{-8}, \\
B_{\pi \beta}^{\text {theo-norm }}=1.040(4)_{\text {stat }}(4)_{\text {syst }} \times 10^{-8},
\end{gathered}
$$

where the statistical (stat), systematic (syst) and $\pi_{e 2}$ normalization uncertainties are separated out. Since 2004 , the $\pi_{e 2}$ branching ratio has become better known, $R_{e / \mu}^{\pi \text {-exp }}=1.2327(23) \times 10^{-4}$ $[1,16]$. This leads to an update of the PiBeta $\pi_{e 3}$ branching ratio result

$$
B_{\pi \beta}^{\text {exp-norm }}=1.038(4)_{\text {stat }}(4)_{\text {syst }}(2)_{\pi_{e 2}} \times 10^{-8}=1.038(6)_{\text {tot }} \times 10^{-8} .
$$

We note that the extraordinary sensitivity of pion beta decay afforded by the SM, with relative uncertainty (excluding the free parameter $V_{u d}$ ) of $\sim 2 \times 10^{-4}$ dominated by the radiative corrections [17], cannot be tested experimentally at the current precision of $\Delta B_{\pi \beta} / B_{\pi \beta} \simeq 0.006$. The same observation applies to the derived value of $V_{u d}$, now updated to

$$
V_{u d}^{\pi \beta}=0.9738(28) \text {, }
$$

which, while in excellent agreement with the PDG average $V_{u d}=0.97370(14)$ [1], is 20 times less precise.

\subsection{Pion radiative electronic decay: $\pi^{+} \rightarrow e^{+} \nu_{e} \gamma$}

In addition to the fundamental physics motivations introduced in Section 24.1 (weak pionic form factors, inputs to LECs, limits on BSM contributions), pion radiative electronic decay generates background events for the pion beta $\left(\pi_{e 3}\right)$ signal, in large enough numbers to require a correction (the reverse also holds). For all these reasons, the PiBeta collaboration has extensively studied the $\pi_{e 2 \gamma}$ decay.

Prior to the early 2000s, data on the $\pi_{e 2 \gamma}$ decay were scarce, and contained significant ambiguities. The doubly differential decay rate $\mathrm{d}^{2} \Gamma_{\pi e 2 \gamma} / \mathrm{d} E_{e} \mathrm{~d} E_{\gamma}$ is separated into structure dependent terms: $S D^{+} \propto\left(F_{A}+F_{V}\right)^{2}, S D^{-} \propto\left(F_{A}-F_{V}\right)^{2}$, the purely-QED IB, and several interference terms of the linear amplitudes, of which the most important are $S_{\text {int }}^{+}$and $S_{\text {int }}^{-}$, the $I B \cdot\left(F_{A}+F_{V}\right)$ and $I B \cdot\left(F_{A}-F_{V}\right)$ terms, respectively. For simplicity in the analysis, dimensionless energy variables are routinely used and are limited to unity: $x, y=2 E_{\gamma, e} / m_{\pi} \in(0,1)$. Since $\left(F_{A}+F_{V}\right)^{2} /\left(F_{A}-F_{V}\right)^{2} \simeq 8, S D^{+}$is the dominant QCD term in the decay. Further, its study is made more accessible by the fact that $S D^{+}$peaks for $y \in(0.9,1)$, and large $x$, where the $I B$ term nearly vanishes. $S D^{-}$, on the other hand, peaks near the diagonal, $x+y=1$, where $I B$ is greatest, and dwarfs $S D^{-}$by several orders of magnitude. Consequently, pre-2000 studies used the conserved vector current (CVC) theoretical value for $F_{V}$ (derived from the $\pi^{0}$ meson lifetime), and reported the ratio $\gamma \equiv F_{A} / F_{V}$ extracted from measurements. Early measurements, along with the inconsistencies and hints of BSM phenomena through a nonzero value for $F_{T}$, the tensor form factor, are discussed in detail in [14]. 

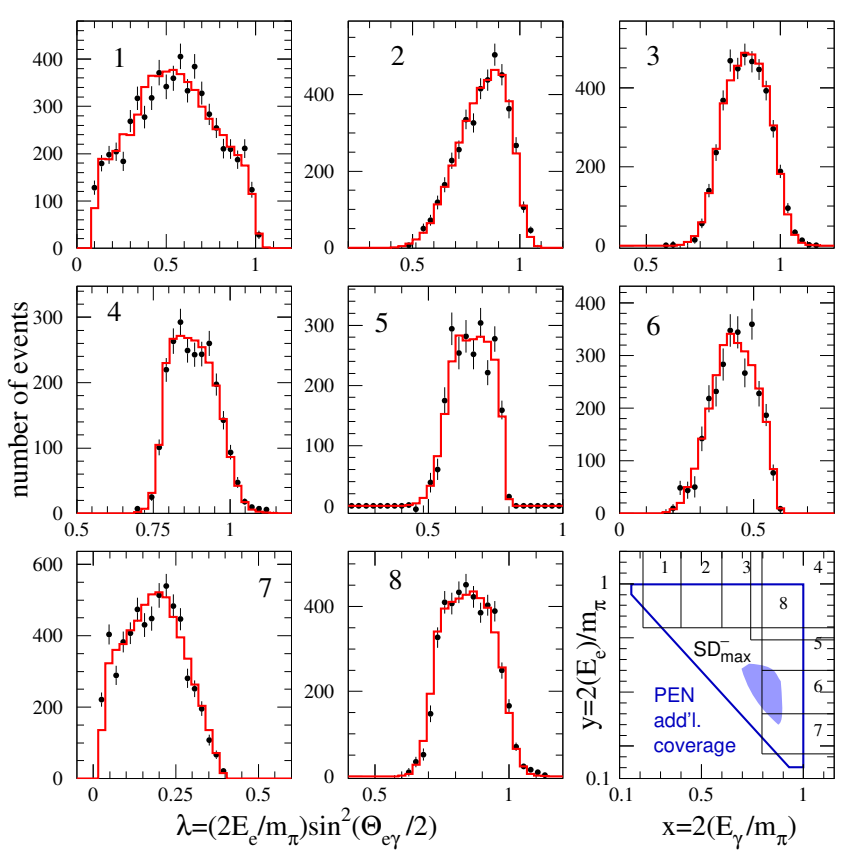

Figure 24.4: Measured (points) and simulated (histogram) values of $\pi_{e 2 \gamma}$ variable $\lambda$ for eight $(x, y)$ regions, mapped in the lower right panel. Triangle: added coverage by PEN [8]. Shaded contour: region of peak relative $S D^{-}$contribution.
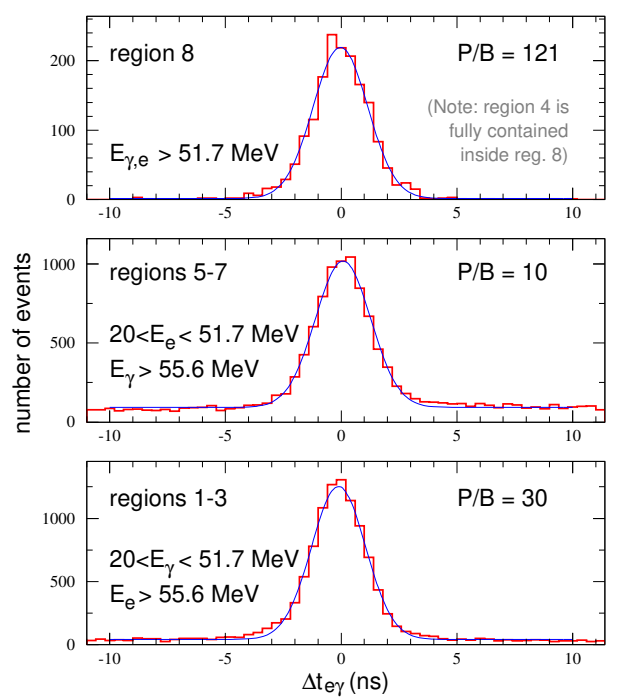

Figure 24.5: Plots of $\Delta t_{e \gamma}$, for three phase space regions in $\pi_{e 2 \gamma}$ decay, defined in Figure 24.4. Accidental background was low for all regions, reflected in the peak to background (P/B) values. Region 4/8 was dominated by Run 1-3 data; Run 4 data dominated the rest.

Against this backdrop, the PiBeta collaboration collected and analyzed over $4 \times 10^{4} \pi_{e 2 \gamma}$ events in Runs 1-3, and published the results in [13]. The precision in $\gamma$ was improved by a factor of four over prior world average, but a significant deficit of events was observed in a region of high $x$ and low $y$. The high beam rate, and trigger configuration during Runs $1-3$, challenged the $\pi_{e 2 \gamma}$ decay systematics in this kinematic regime.

Given the above, in 2004 the PiBeta collaboration carried out Run 4 at much lower beam rate $\left(\sim 10^{5} \pi_{\text {stop }} / \mathrm{s}\right)$, focused on low-threshold $\pi_{e 2 \gamma}$ events. This made possible a precise calibration of subtle calorimeter gain differences in the low- and high-threshold triggers, the key to resolving previously observed inconsistencies. Results of the combined Run 1-4 data set analysis, with over $6.5 \times 10^{4} \pi_{e 2 \gamma}$ events, were published in [18]. Kinematic coverage is shown in Figure 24.4, while Figure 24.5 illustrates the low level of accidental background present in the $e^{+}-\gamma$ time difference data. Data in Figure 24.4 are presented in terms of $\lambda=y \sin ^{2}\left(\theta_{e \gamma} / 2\right)$, where $\theta_{e \gamma}$ is the reconstructed $e^{+}-\gamma$ opening angle. (Unlike $y, \lambda$ retains the constant $0-1$ value range regardless of $x$.) Agreement with the simulation based on best-fit values for $F_{A}$ and $F_{V}$ is excellent in all regions.

Contours of the best-fit values for $F_{A}$ and $F_{V}$ are shown in Figure 24.6. The thin shape of the resulting ellipse reflects the $\sim 1 \%$ precision of the measurement of $F_{A}+F_{V}\left(S D^{+}\right.$term), and the much lower sensitivity to $F_{A}-F_{V}$, i.e., $S D^{-}$. The narrow linear dependence of $F_{A}$ on $F_{V}$ reported in [18] enables future updates of the best-fit value of $F_{A}$ based on improved evaluations of $F_{V}^{\mathrm{CVC}}$.

Figure 24.6 also plots $a$, the slope parameter of $F_{V}$ with respect to the momentum transfer to the lepton pair $q_{e v}^{2}$, a first such result, made possible by the broad combined kinematic coverage of PiBeta Runs 1-4. The slope is in qualitative agreement with the $\chi$ PT calculation of Mateu and Portoles [19]. 


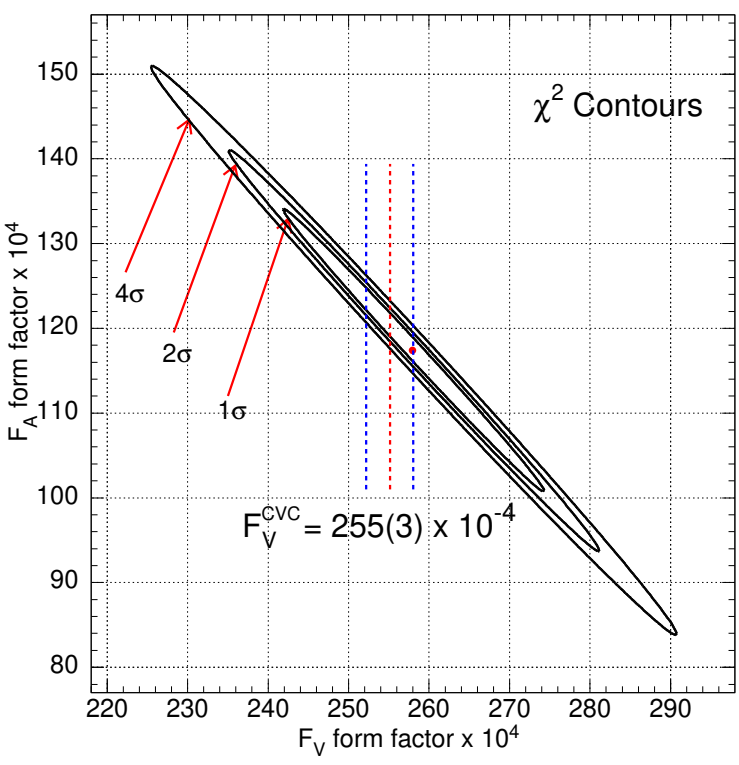

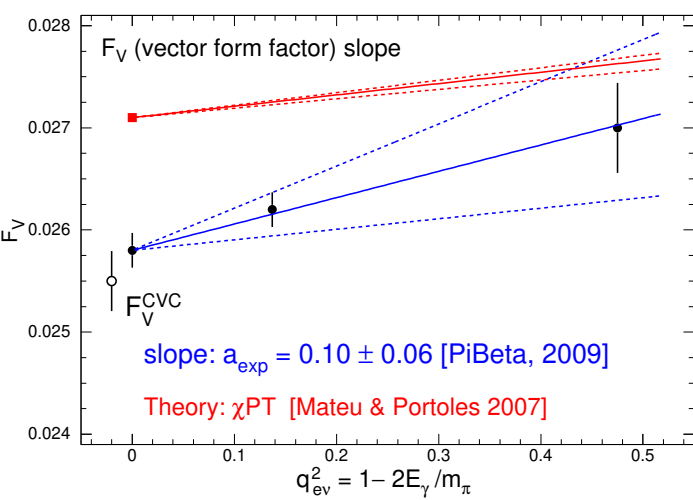

Figure 24.6: $\quad F_{A}$ and $F_{V}$ best fit contours for the full PiBeta $\pi_{e 2 \gamma}$ data set (left). Corresponding $q_{e v}^{2}$ dependence of $F_{V}$ (above). The CVC value for $F_{V}$ is plotted in both.

Analysis of the integral $\pi_{e 2 \gamma}$ decay rate yielded the primary result: branching ratio for the kinematic region $E_{\gamma}>10 \mathrm{MeV}$ and $\theta_{e \gamma}>40^{\circ}$ of $B^{\exp }=73.86(54) \times 10^{-8}$. At $<1 \%$, this result marked a $\sim 20$-fold precision improvement over previous measurements [1]. The excellent fit of the $\pi_{e 2 \gamma}$ differential decay rates has led to the arguably most important result of this work, the limit on a possible admixture of the tensor interaction $-5.2 \times 10^{-4}<F_{T}<4.0 \times 10^{-4}$ with $90 \%$ confidence [18]. To date, this limit provides the strongest constraint on a possible BSM tensor coupling [20].

\subsection{Conclusions and path forward}

The PiBeta research program has produced an order of magnitude improvement in the precision of the $\pi_{e 3}$ and $\pi_{e 2 \gamma}$ branching ratios, and related SM observables, low energy QCD parameters (LECs), and a leading limit on BSM tensor coupling.

PEN, the successor experiment to PiBeta, has focused on $\pi_{e 2}$ decay [8], and expanded the $\pi_{e 2 \gamma}$ kinematic coverage (Figure 24.4), fully enclosing the region of peak $S D^{-} /$total relative yield $^{2}$. This is a modest improvement. A new, dedicated experiment would be needed to achieve greater sensitivity.

The scientific case is mounting for a new generation of experiment to fully exploit the precision of the SM description of pion decays, and realize the potential to settle the decadesold question of CKM unitarity in a process free from complex nuclear structure corrections.

\section{Acknowledgments}

This work has been supported by the U.S. National Science Foundation, the Paul Scherrer Institute, and the Russian Foundation for Basic Research.

\section{References}

[1] P. Zyla et al., Review of Particle Physics, PTEP 2020(8), 083 C01 (2020), doi:10.1093/ptep/ptaa104.

\footnotetext{
${ }^{2}$ Even at its peak relative to other terms, $S D^{-}$locally contributes only $\sim 8 \%$ of the decays.
} 
[2] J. Hardy and I. Towner, Superallowed $0^{+} \rightarrow 0^{+}$nuclear $\beta$ decays: 2020 critical survey, with implications for $V_{u d} \ldots$, Phys. Rev. C 102(4), 045501 (2020), doi:10.1103/PhysRevC.102.045501.

[3] A. Czarnecki, W. Marciano and A. Sirlin, Pion beta decay and Cabibbo-Kobayashi-Maskawa unitarity, Phys. Rev. D 101(9), 091301 (2020), doi:10.1103/PhysRevD.101.091301, 1911.04685.

[4] B. Märkisch et al., Measurement of the weak axial-vector coupling constant in the decay of free neutrons using ..., Phys. Rev. Lett. 122(24), 242501 (2019), doi:10.1103/PhysRevLett.122.242501, 1812.04666.

[5] A. Czarnecki, W. Marciano and A. Sirlin, Neutron lifetime and axial coupling connection, Phys. Rev. Lett. 120(20), 202002 (2018), doi:10.1103/PhysRevLett.120.202002, 1802. 01804.

[6] S. Baeßler, J. Bowman, S. Penttilä and D. Počanić, New precision measurements of free neutron beta decay with cold neutrons, J. Phys. G 41, 114003 (2014), doi:10.1088/0954$3899 / 41 / 11 / 114003,1408.4737$.

[7] Pibeta experiment home page, http://pibeta.phys.virginia.edu.

[8] D. Počanić, Pion electronic decay and lepton universality, this volume of SciPost Phys. Proc., paper 025 (2021).

[9] P. De Baenst and J. Pestieau, Extension of Cabibbo's theory to radiative leptonic decays of pseudoscalar mesons, Nuovo Cim. A 53, 407 (1968), doi:10.1007/BF02800120.

[10] D. Bryman, P. Depommier and C. Leroy, $\pi \rightarrow e v, \pi \rightarrow e v \gamma$ decays and related processes, Phys. Rept. 88, 151 (1982), doi:10.1016/0370-1573(82)90162-4.

[11] J. Donoghue, E. Golowich and B. Holstein, Dynamics of the standard model, Cambridge University Press, Cambridge, doi:10.1017/CBO9780511524370 (1992).

[12] J. Bijnens and G. Ecker, Mesonic low-energy constants, Ann. Rev. Nucl. Part. Sci. 64, 149 (2014), doi:10.1146/annurev-nucl-102313-025528, 1405.6488.

[13] E. Frlež et al., Design, commissioning and performance of the PIBETA detector at PSI, Nucl. Instrum. Meth. A 526, 300 (2004), doi:10.1016/j.nima.2004.03.137, hep-ex/0312017.

[14] D. Počanić, E. Frlež and A. van der Schaaf, Experimental study of rare charged pion decays, J. Phys. G 41, 114002 (2014), doi:10.1088/0954-3899/41/11/114002, 1407.2865.

[15] D. Počanić et al., Precise measurement of the $\pi^{+} \rightarrow \pi^{0} e^{+} v$ branching ratio, Phys. Rev. Lett. 93, 181803 (2004), doi:10.1103/PhysRevLett.93.181803, hep-ex/0312030.

[16] A. Aguilar-Arevalo et al., Improved measurement of the $\pi \rightarrow$ ev branching ratio, Phys. Rev. Lett. 115(7), 071801 (2015), doi:10.1103/PhysRevLett.115.071801, 1506.05845.

[17] A. Czarnecki, W. J. Marciano and A. Sirlin, Radiative corrections to neutron and nuclear beta decays revisited, Phys. Rev. D 100(7), 073008 (2019), doi:10.1103/PhysRevD.100.073008, 1907.06737.

[18] M. Bychkov et al., New precise measurement of the pion weak form factors in $\pi^{+} \rightarrow e^{+} v \gamma$ decay, Phys. Rev. Lett. 103, 051802 (2009), doi:10.1103/PhysRevLett.103.051802, 0804.1815. 
[19] V. Mateu and J. Portoles, Form-factors in radiative pion decay, Eur. Phys. J. C 52, 325 (2007), doi:10.1140/epjc/s10052-007-0393-5, 0706.1039.

[20] T. Bhattacharya et al., Probing novel scalar and tensor interactions from (ultra)cold neutrons to the LHC, Phys. Rev. D 85, 054512 (2012), doi:10.1103/PhysRevD.85.054512, 1110.6448 . 